$10,12,09$

\title{
Экспериментальное исследование динамики решетки индивидуальных полупроводниковых двустенных углеродных нанотрубок: тангенциальные $G$-моды
}

\author{
() Д.И. Левшов ${ }^{1}$, H.N. $\operatorname{Tran}^{2}$, Ю.С. Слабодян ${ }^{1}$, А.В. Осадчий ${ }^{3,4}$, С.Б. Рошаль ${ }^{1}$, Ю.И. Юзюк ${ }^{1}$ \\ ${ }^{1}$ Южный фредеральный университет, \\ Ростов-на-Дону, Россия \\ ${ }^{2}$ Laboratoire Charles Coulomb UMR 5221, Université de Montpellier, CNRS, \\ Montpellier, France \\ ${ }^{3}$ Институт общей фозики им. А.М. Прохорова РАН, \\ Москва, Россия \\ ${ }^{4}$ Национальный исследовательский ядерный университет „МИФИ“, \\ Москва, Россия \\ E-mail: dmitry.levshov@gmail.com
}

(Поступила в Редакцию 4 июля 2016 г.)

\begin{abstract}
Проведены исследования тангенциальных $G$-мод индивидуальных полупроводниковых двустенных углеродных нанотрубок методом резонансной спектроскопии комбинационного рассеяния света в широком диапазоне длин волн лазерного возбуждения. Индивидуальные подвешенные нанотрубки синтезированы методом химического осаждения из парогазовой фазы. Индексы хиральности $(n, m)$ нанотрубок определялись методами электронной дифракции и просвечивающей электронной микроскопии высокого разрешения. В спектрах комбинационного рассеяния двустенных нанотрубок наблюдается существенный сдвиг тангенциальных мод в сравнении с аналогичными модами одностенных нанотрубок. Показано, что величина сдвига зависит от межслойного расстояния и от величины ван-дер-ваальсова взаимодействия между слоями двустенной нанотрубки.
\end{abstract}

Исследование выполнено за счет гранта Российского фонда фундаментальных исследований (проект № 15-02-08340).

DOI: 10.21883/FTT.2017.02.44057.274

\section{1. Введение}

Двустенные углеродные нанотрубки (ДУНТ) представляют собой наноструктуры, сформированные из двух коаксиальных углеродных слоев. Их промежуточное положение между одностенными и многостенными трубками вызывает повышенный интерес со стороны научного сообщества как с фундаментальной точки зрения (ДУНТ являются простейшими системами для изучения ван-дер-ваальсового взаимодействия), так и с прикладной (возможность функционализировать внешнюю УНТ при сохранении свойств внутренней открывает ряд интересных возможностей в индустрии и технике) [1-3].

Изучение физических свойств нанотрубок традиционно проводится с помощью методов оптической спектроскопии. Среди них наиболее популярным и эффективным является спектроскопия комбинационного рассеяния света (КРС) [4]. В спектрах КРС может наблюдаться целый ряд активных мод, среди которых особое место занимают тангенциальные $G$-моды. Данные моды соответствуют колебаниям атомов, направленным по касательной к поверхности нанотрубки. В зависимости от направления колебаний $G$-моды разделяют на продольные (LO) и поперечные (TO). Известно, что характерные особенности $G$-мод (профиль линии, частота, полуширина, относительная интенсивность) крайне чувствительны, с одной стороны, к собственным структурным параметром ОУНТ (диаметру $d$, хиральному углу $\theta$, типу проводимости), а с другой стороны, к различным внешним воздействиям: к допированию [5], напряжению и давлению $[6,7]$, и изменению в окружении нанотрубки [8]. Следует отметить, что подробная информация о $G$-модах была получена в основном из измерений спектров КРС, выполненных на пространственно изолированных индивидуальных структурноидентифицированных ОУНТ [9]. Это связано с тем, что в пучках ОУНТ или в растворах индивидуализированных ОУНТ широкий разброс нанотрубок по диаметру и хиральностям приводит к перекрытию $G$-мод и, как следствие невозможности однозначной интерпретации экспериментальных данных. Изучение характерных особенностей $G$-мод на основе макро образцов стало возможно относительно недавно и только после разработки методов сортировки нанотрубок по определенной хиральности [10].

Важно отметить, что однозначного исследования тангенциальных мод структурно-идентифицированных двустенных нанотрубок до настоящего момента не проводилось. Хотя ранее и было проведено изучение индивидуальных полупроводниковых ДУНТ расположенных на подложке [11-13], в этих работах структуру двустенных нанотрубок идентифицировали косвенным способом с 


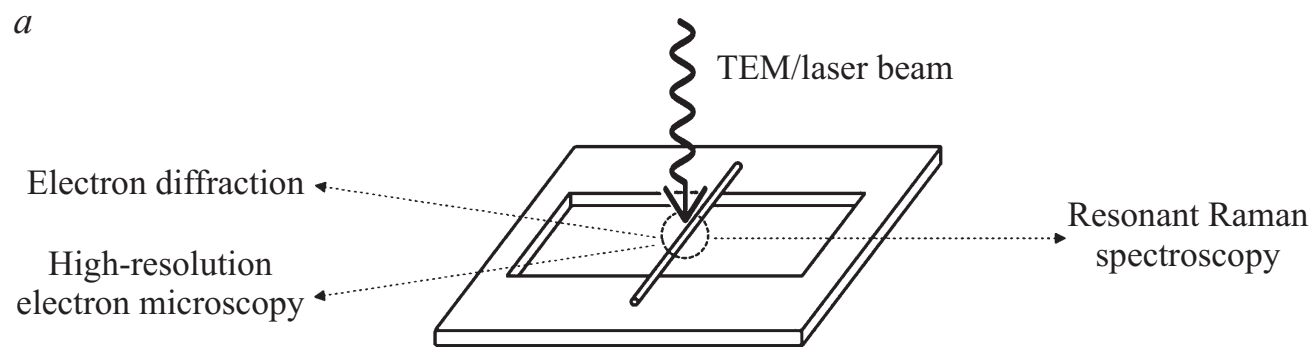

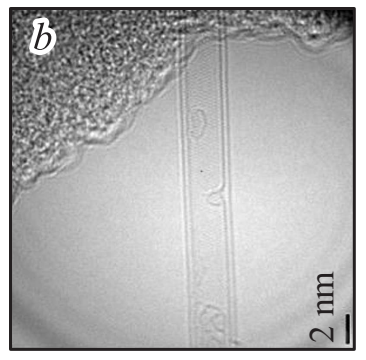

HRTEM

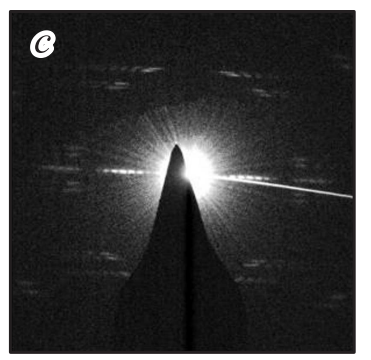

EDP

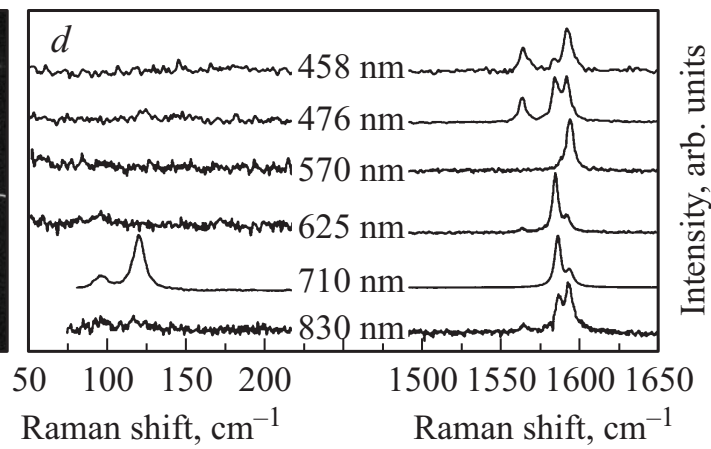

Рис. 1. a) Схематическое изображение экспериментальной методики, использованной для изучения индивидуальных двустенных УНТ; примеры получаемых экспериментальных данных, в частности: $b$ ) электронное изображение, $c$ ) электронная дифрактограмма и $d)$ спектры КРС двустенной углеродной нанотрубки $(22,11) @(27,17)$. Спектры нормализованы по интенсивности $G$-моды для иллюстративных целей.

помощью сравнения экспериментальных резонансных энергий с теоретически рассчитанными энергиями переходов. Одним из результатов вышеописанных работ стало установление факта, что частота $G$-компоненты чувствительна к межслойному взаимодействию.

В данной работе представлено исследование тангенциальных мод индивидуальных структурно-идентифицированных ДУНТ в зависимости от межслойного расстояния. Независимое определение структурных параметров ДУНТ методами электронной дифракции и просвечивающей электронной микроскопии позволило нам однозначно интерпретировать наблюдаемые особенности спектров КРС двустенных нанотрубок. В результате исследований экспериментально обнаружен сдвиг тангенциальных мод ДУНТ по сравнению с соответствующими модами одностенных изолированных слоев. Показано, что величина сдвига зависит от межслойного расстояния и от величины эффективного давления внутри двустенной нанотрубки.

\section{2. Особенности методики исследований}

Для однозначной интерпретации экспериментальных данных в данной работе был использован метод комбинации резонансной спектроскопии КРС, электронной дифракции и просвечивающей электронной микроскопии (рис. 1,a). Двустенные индивидуальные горизонтально- ориентированные углеродные нанотрубки были получены для исследований методом каталитического химического осаждения из парогазовой фазы (CCVD), который был ранее описан в работах [14-21]. Рост ДУНТ происходил либо на специально подготовленных кремниевых подложках со сквозными траншеями (см. схематичное изображение на рис. $1, a$; типичные размеры отверстий составляли $50-100 \mu$ в ширину и до $1500 \mu$ в длину), либо на коммерческих перфорированных мембранах SPI для HRTEM исследований (размер отверстий в этом случае составлял $3 \mu$ ).

Такая методика изготовления образцов позволила нам, во-первых, исследовать собственные свойства ДУНТ в подвешенных областях, где отсутствует взаимодействие нанотрубки и подложки, и во-вторых, проводить на одних и тех же ДУНТ как эксперименты по электронной дифракции и электронной микроскопии, так и эксперименты по спектроскопии КРС. С одной стороны, атомная структура нанотрубок определялась электронными методами напрямую из электронных изображений и электронных дифрактограмм без привлечения различных моделизаций, сопутствующих оптическим подходам. С другой стороны, независимое определение геометрического строения нанотрубок позволило нам однозначно связать наблюдаемые особенности КРС-активных мод с теми или иными структурными параметрами ДУНТ.

Эксперименты по электронной дифракции и электронной микроскопии проводились на микроскопе FEI Titan Cubed Cs corrected 60-300 kV TEM при напря- 
Структурные параметры шести полупроводниковых ДУНТ, полученные из экспериментов по электронной дифракции. Нанотрубки приведены в порядке увеличения межслойного расстояния $\delta r$

\begin{tabular}{c|c|c|c|c|c|c}
\hline № & Chiral indices & $\begin{array}{c}\text { Diameter } \\
(\mathrm{nm})\end{array}$ & $\begin{array}{c}\text { Chiral angle, } \\
\circ\end{array}$ & $\begin{array}{c}\text { Type } \\
2 n+m\end{array}$ & $\begin{array}{c}\delta r \\
(\mathrm{~nm})\end{array}$ & $\begin{array}{c}\text { Effective pressure } \\
(\mathrm{GPa})\end{array}$ \\
\hline 1 & $(12,8) @(16,14)$ & $1.366 @ 2.037$ & $23.41 @ 27.8$ & $2 @ 1$ & 0.335 & 0.5 \\
2 & $(16,12) @(27,10)$ & $1.905 @ 2.595$ & $25.28 @ 15.14$ & $1 @ 2$ & 0.345 & -0.5 \\
3 & $(10,9) @(18,11)$ & $1.289 @ 1.985$ & $28.26 @ 22.07$ & $2 @ 2$ & 0.348 & -0.8 \\
4 & $(20,4) @(20,16)$ & $1.745 @ 2.447$ & $8.95 @ 26.33$ & $2 @ 2$ & 0.351 & -1 \\
5 & $(13,9) @(24,7)$ & $1.501 @ 2.206$ & $24.01 @ 12.43$ & $2 @ 1$ & 0.353 & -1.1 \\
6 & $(22,11) @(27,17)$ & $2.280 @ 3.011$ & $19.11 @ 22.52$ & 192 & 0.365 &
\end{tabular}

жении $80 \mathrm{kV}$. Электронные дифрактограммы записывались с использованием CCD-детектора (Gatan Ultrascan $2 \mathrm{~K} \times 2 \mathrm{~K})$ с двумя типами камер: 285 и $460 \mathrm{~mm}$. Время накопления электронных дифрактограмм не превышало $10 \mathrm{~s}$. Особое внимание уделялось тому, чтобы электронный пучок не повреждал нанотрубки. Наконец, определение структурных параметров УНТ проводилось согласно существующим стандартным процедурам [22].

Эксперименты по спектроскопии резонансного КРС проводились на спектрометрах Jobin Yvon T64000, iHR 550 Jobin Yvon и Renishaw InVia Reflex. Для получения максимальной спектральной информации спектры КРС углеродных нанотрубок измерялись в широком диапазоне длин волн лазерного возбуждения: 458, 476, 488, 514, 532, 568-633, 647, 676 и 710-900 nm (дискретные лазеры $\mathrm{Ar}^{+}, \mathrm{Kr}^{+}, \mathrm{He}-\mathrm{Ne}$ и лазеры с перестраиваемой длиной волны Ti:sapphire и Dye laser). Рассеянный свет собирался с использованием $100 \times$ объектива (N.A. $=0.95)$ в конфигурации обратного рассеяния. Во всех измерениях поляризации падающего и рассеянного света были параллельны оси нанотрубки ( $\|$-геометрия). Чтобы избежать нагревания нанотрубок, мощность лазерного излучения поддерживалась на уровне $50 \mu \mathrm{W}$. Параметры (частота, интенсивность, полуширина, интегральная площадь) основных КРС-активных линий были получены в результате моделирования спектров функциями Лоренца.

\section{3. Результаты и обсуждение}

На рис. $1, b$ и $c$ представлены электронное изображение и электронная дифрактограмма одной из типичных полупроводниковых двустенных углеродных нанотрубок, исследованных в данной работе. Во-первых, по электронному изображению можно явно установить двустенный тип нанотрубки. Во-вторых, анализ электронных дифрактограмм, выполненный согласно существующим в литературе методикам [22], позволил определить индексы хиральности данной ДУНТ как $(22,11) @(27,17)$. Структурные параметры других индивидуальных двустенных нанотрубок, полученные аналогичным образом, представлены в таблице. В част- ности, в таблице отмечены диаметры слоев $d_{i n} @ d_{\text {out }}$, хиральные углы $\theta_{\text {in }} @ \theta_{\text {out }}$, тип проводимости, рассчитанный по формуле $(2 n+m) \bmod 3$, межслойное расстояние $\delta r=\left(d_{\text {out }}-d_{\text {in }}\right) / 2$. Отметим, что представленные в данной работе ДУНТ имеют значения межслойного расстояния $\delta r$ в диапазоне от 0.335 до $0.365 \mathrm{~nm}$. Средний диаметр ДУНТ варьируется от 1.637 до $2.644 \mathrm{~nm}$.

На рис. 1, $d$ показаны спектры КРС двустенной углеродной нанотрубки $(22,11) @(27,17)$, измеренные на длинах волн лазерного возбуждения 458, 476, 570, 625,710 и $830 \mathrm{~nm}$. Отметим, что спектры измерялись с тех же областей УНТ, с которых ранее были получены электронные дифрактограммы и электронные изображения. Изменения числа наблюдаемых линий и их относительной интенсивности в зависимости от используемой длины волны лазерного возбуждения подтверждают резонансный характер КРС нанотрубок. В низкочастотной области (например, при длине волны возбуждения $710 \mathrm{~nm}$ ) наблюдается две линии с частотами 98 и $121 \mathrm{~cm}^{-1}$, которые относятся к синфазной и противофазной радиальным дыхательно-подобным модам (RBLM) соответственно. C другой стороны, при длине волны возбуждения $476 \mathrm{~nm}$ в высокочастотной области спектров КРС наблюдается три компоненты с частотами 1562,1583 и $1592 \mathrm{~cm}^{-1}$.

Необходимо отметить, что положение высокочастотных пиков не соответствует частотам тангенциальных мод, которые можно было бы ожидать для составляющих ДУНТ одностенных слоев. Например, согласно ранее установленным зависимостям частоты $G$-моды от диаметра одностенных нанотрубок $[10,23,24]$ в ОУНТ $(22,11)$ с диаметром $2.278 \mathrm{~nm}$ TO- и LO-моды имеют частоты, равные 1574 и $1591 \mathrm{~cm}^{-1}$ соответственно. А в ОУНТ $(27,17)$ с диаметром $3.009 \mathrm{~nm}$ соответственно 1579 и $1589 \mathrm{~cm}^{-1}$.

Однозначное присвоение наблюдаемых в спектрах ДУНТ $(22,11) @(27,17)$ линий к соответствующим слоям стало возможно благодаря использованию широкого набора длин волн возбуждения и систематическому измерению профилей резонанса радиальных и тангенциальных мод. К примеру, на рис. 2 представлены профили резонанса радиальных дыхательно-подобных мод (слева) и тангенциальных $G$-мод (справа) в диапазоне энергий возбуждения $1.9-2.2 \mathrm{eV}$ для ДУНТ $(22,11) @(27,17)$. 


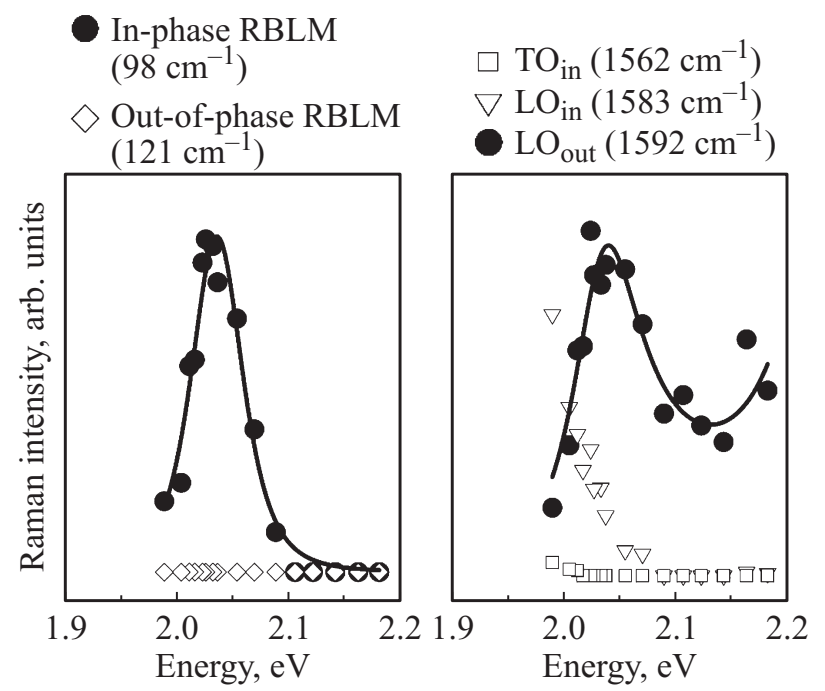

Рис. 2. Профили резонанса радиальных дыхательно-подобных (RBLM,слева) и тангенциальных ( $G$-моды, справа) мод двустенной углеродной нанотрубки $(22,11) @(27,17)$.

Интенсивность мод как функция энергии возбуждения лазера $\left(E_{L}\right)$ задается выражением

$$
I\left(E_{L}\right) \propto \frac{1}{\left[\left(E_{L}-E_{i i}\right)^{2}+\frac{\Gamma^{2}}{4}\right] \cdot\left[\left(E_{L}-E_{i i}+E_{\mathrm{phonon}}\right)^{2}+\frac{\Gamma^{2}}{4}\right]},
$$

где $\Gamma$ - полуширина, а $E_{i i}$ и $E_{\text {phonon }}-$ энергия электронного перехода и энергия соответствующего фонона.

Моделирование резонансных профилей с использованием формулы (1), где $E_{i i}$ и $\Gamma$ взяты в качестве варьируемых параметров, показало, что синфазная радиальная мода $\left(98 \mathrm{~cm}^{-1}\right)$ и расположенная на $1592 \mathrm{~cm}^{-1}$ $G$-мода имеют одну и ту же энергию резонанса $\left(E_{33 \exp }=2.05 \mathrm{eV}\right)$. С другой стороны, линии с частотами 1562 и $1583 \mathrm{~cm}^{-1}$ находятся в резонансе на тех же энергиях, что и противофазная радиальная мода (ясно видно из рис. $1, d$ при длине волны $710 \mathrm{~nm}$ ). Подобное поведение систематически обнаруживается и в других ДУНТ из таблицы. Так как ранее было установлено $[16,25]$, что синфазные и противофазные радиальные моды относятся к внешнему и внутреннему слоям соответственно, мы также можем предположить, что $G$-мода с частотой $1592 \mathrm{~cm}^{-1}$ относится к внешнему слою, а с частотами 1562 и $1583 \mathrm{~cm}^{-1}$ - к внутреннему. Такое отнесение линий позволяет однозначно констатировать тот факт, что в ДУНТ частоты $G$-мод заметно сдвинуты по сравнению с $G$-модами соответствующих одностенных УНТ.

Выше описанный эффект более отчетливо виден на рис. 3, где показана зависимость тангенциальных мод УНТ как функция диаметра. Открытыми символами представлены экспериментальные данные, полученные на индивидуальных подвешенных ОУНТ [24,26] (открытые треугольники) и на суспензиях индивидуальных
ОУНТ [10] (открытые кружки). Сплошной и пунктирной линиями показаны зависимости частот LO- и TO-мод от диаметра ОУНТ по формуле, предложенной в работе [10]. Черными символами представлены частоты TO- и LO-мод внутренних слоев ДУНT, изученных в данной работе. Ясно видно, что частоты тангенциальных мод внутренних слоев сдвинуты по отношению к ОУНТ, однако величина сдвига сильно варьируется от одной ДУНТ к другой.

Тщательный анализ экспериментальных данных позволил нам обнаружить связь между величиной сдвига тангенциальных мод и межслойным расстоянием в ДУНТ. В качестве примера наблюдаемого эффекта на рис. 4 показаны спектры $G$-мод, измеренные на трех типичных индивидуальных полупроводниковых ДУНТ из таблицы, имеющих различное межслойное расстояние. Спектр нанотрубки $(12,8) @(16,14)$, имеющей $\delta r=0.335 \mathrm{~nm}$, представляет собой простую сумму $G$-мод составляющих ее индивидуальных ОУНТ (наблюдаемый на рис. 4 сдвиг частот TO $\left(0.5 \mathrm{~cm}^{-1}\right)$ - и LO $\left(1 \mathrm{~cm}^{-1}\right)$-мод сопоставим с погрешностью измерения). В противоположность этому, $G$-моды внутренних слоев в ДУНТ $(13,9) @(24,7)$ с $\delta r=0.353$ и в ДУНТ $(22,11) @(27,17)$ с $\delta r=0.365$ сдвинуты значительно больше (вплоть до $13 \mathrm{~cm}^{-1}$, см. рис. 4) по отношению к LO- и TO-модам соответствующих одностенных нанотрубок. Общая наблюдаемая тенденция следующая: чем больше межслойное расстояние в ДУНТ, тем больше сдвиг тангенциальных мод внутреннего слоя в область низких частот.

Для объяснения наблюдаемых сдвигов частот тангенциальных мод оценим силу ван-дер-ваальсового взаимодействия в изучаемых двустенных нанотрубках как функцию межслойного расстояния. Ранее, численные оценки

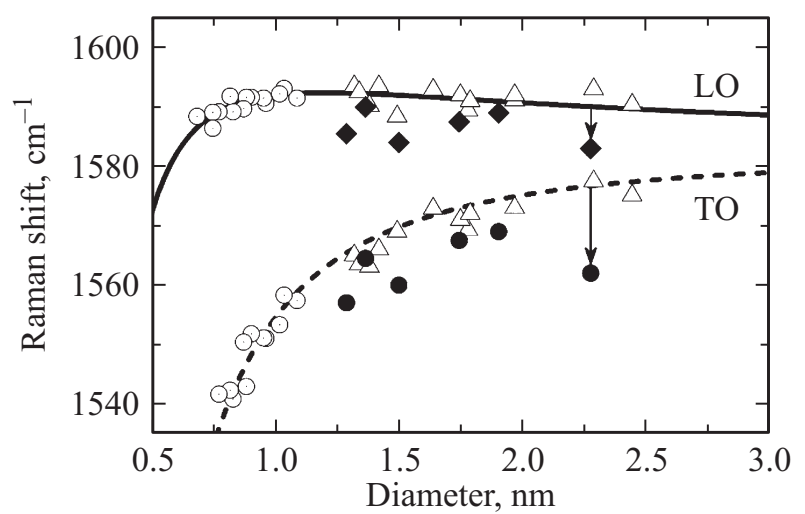

Рис. 3. Зависимости частот LO (сплошная линия) и TO (пунктирная линия) мод от диаметра одностенной нанотрубки [10]. Открытыми символами представлены экспериментальные данные, полученные на индивидуальных подвешенных ОУНТ $[24,26]$ (открытые треугольники) и на суспензиях индивидуальных ОУНТ [10] (открытые кружки). Черными символами представлены частоты TO- и LO-мод внутренних слоев ДУНТ, изученных в данной работе. Сдвиг тангенциальных мод ДУНТ $(22,11) @(27,17)$ по сравнению с одностенными ОУНТ показан стрелками. 


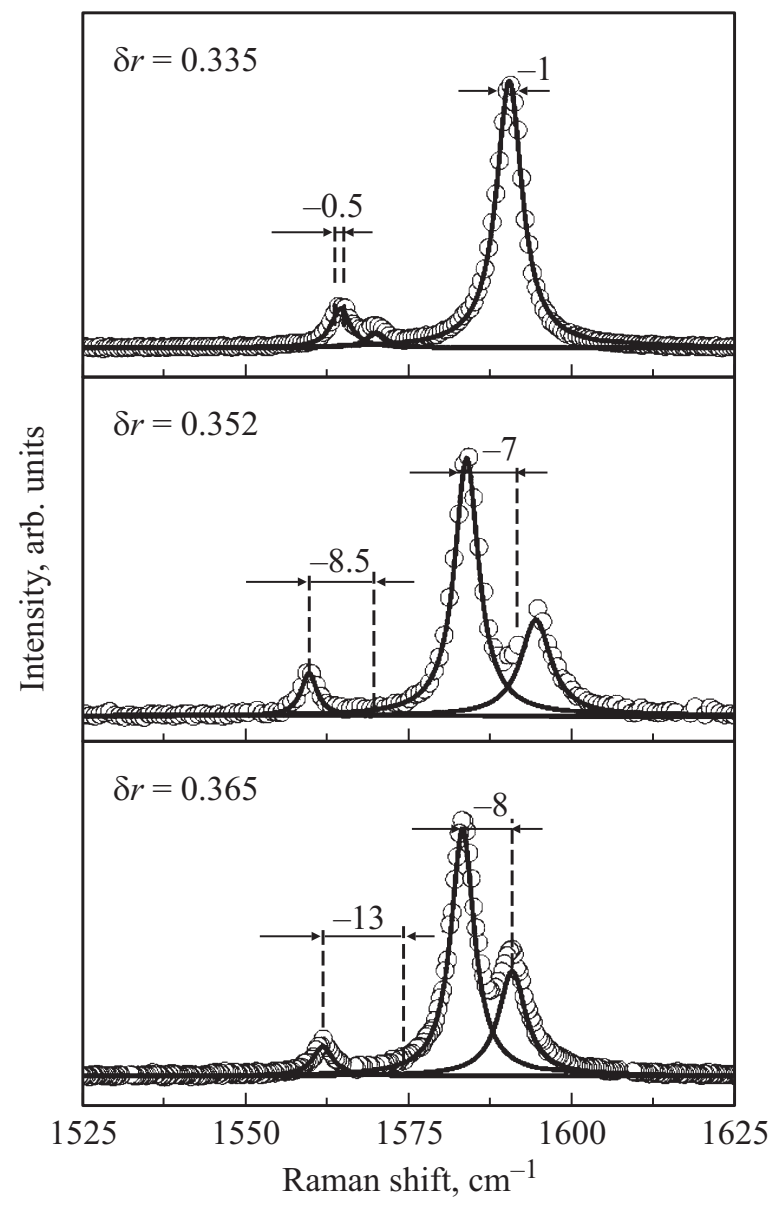

Рис. 4. Сравнение $G$-мод, измеренных на дУНТ $(12,8) @(16,14),(13,9) @(24,7)$ и $(22,11) @(27,17)$. Межслойное расстояние увеличивается сверху вниз $(0.335,0.352$, $0.365 \mathrm{~nm})$. Стрелки и пунктирные линии показывают сдвиг частот LO- и ТО-мод ДУНТ по сравнению с соответствующими индивидуальными одностенными УНТ. Спектры аппроксимированы с помощью функций Лоренца.

ван-дер-ваальсова взаимодействия между слоями ДУНТ были проведены на основе радиальных дыхательноподобных мод [25]. В частности, была оценена силовая константа $\left(k_{c}\right)$ ван-дер-ваальсова взаимодействия между внутренней и внешней нанотрубками [25]. Было установлено, что радиальные колебания одностенных слоев в ДУНТ не являются независимыми и для их корректного описания необходимо использовать модель связанных гармонических колебаний. Сравнив значения силовых констант $k_{c}$ с измерениями, выполненными на гексагональном графите [27], авторы обнаружили связь между межслойным расстоянием и внутренним эффективным давлением в двустенной нанотрубке (см. рис. $3, d$, работы [25]). Liu и соавторы обнаружили, что при $\delta r>0.34 \mathrm{~nm}$ слои ДУНТ испытывают отрицательное гидростатическое давление. Отрицательное (положительное) давление при ван-дер-ваальсовом взаимодействии соответствует небольшому сжатию (расширению) внешнего слоя и одновременному расширению (сжатию) внутреннего слоя (см. также [28]).

Аналогичным образом для всех исследуемых в данной работе индивидуальных ДУНТ в рамках модели связанных гармонических осцилляторов мы рассчитали силовые константы $k_{c}$ ван-дер-ваальсового взаимодействия, а также эффективное давление внутри нанотрубки, как функцию межслойного расстояния. Во-первых, мы обнаружили хорошее согласие между нашими данными и данными работы Liu и соавторов. Во-вторых, наши расчеты показали, что в исследуемых ДУНТ, имеющих межслойное расстояние в диапазоне $0.335-0.365 \mathrm{~nm}$, эффективное давление изменяется в пределах от +0.5 до $-1.8 \mathrm{GPa}$. К примеру, в ДУНТ $(13,9) @(24,7)$ с $\delta r=0.352 \mathrm{~nm}$ существует давление, равное $-1.1 \mathrm{GPa}$ (см. последнюю колонку таблицы).

Проведем независимую оценку эффекта давления на тангенциальные моды. Хорошо известно, что колебательные моды углеродных нанотрубок зависят от гидростатического давления. К примеру, Лебедкин и соавторы [7] изучили зависимость от давления основных КРС-активных $G$-мод нанотрубок типа НiPCO, диспергированных в воде. Они обнаружили, что зависимость частот TO- и LO-мод одностенных углеродных нанотрубок от гидростатического давления составляет $6.5-8 \mathrm{~cm}^{-1} / \mathrm{GPa}$. Итак, в нашем случае в двустенной УНT $(13,9) @(24,7)$, TO- и LO-моды слоя $(13,9)$ имеют частоты 1560 и $1584 \mathrm{~cm}^{-1}$, соответственно. В то время как в изолированной ОУНТ $(13,9)$ ТО и LO моды имеют частоты 1569 и $1591 \mathrm{~cm}^{-1}$. То есть сдвиг частот составляет 9 и $7 \mathrm{~cm}^{-1}$ соответственно. Экстраполировав данные работы Лебедкина в область отрицательного гидростатического давления, мы обнаруживаем, что сдвиг в $7-9 \mathrm{~cm}^{-1}$ для TO- и LO-мод внутреннего слоя должен соответствовать отрицательному давлению, равному $\approx 1.2 \mathrm{GPa}$. Эта оценка хорошо согласуется со

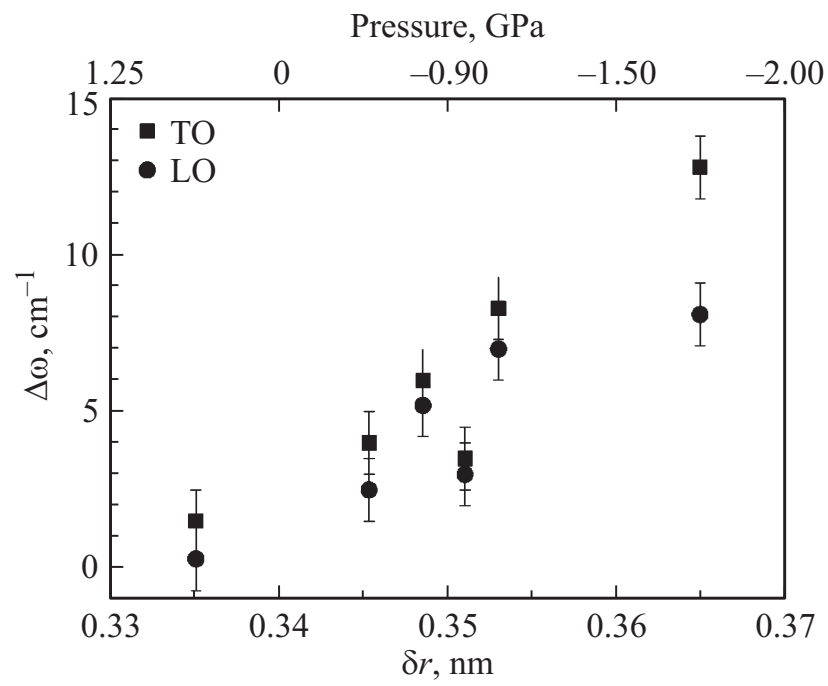

Рис. 5. Зависимость сдвига тангенциальных TO- и LO-мод внутренних слоев двустенных нанотрубок от межслойного расстояния и эффективного давления. 
значением давления, которое было выше получено на основе анализа радиальных дыхательно-подобных мод [25].

Итак, все наблюдаемые зависимости находятся в хорошем согласии с концепцией отрицательного давления внутри двустенной нанотрубки (см. рис. 5). Действительно, при отрицательном давлении происходит небольшое сжатие внешнего слоя и одновременное расширение внутреннего [28]. Возникающее таким образом растяжение (сокращение) С-С-связей во внутреннем (внешнем) слое объясняет уменьшение (увеличение) частоты его $G$-мод. Очевидно, что при положительном давлении ожидается обратное поведение $G$-мод внутреннего (сдвиг в область высоких частот) и внешнего (сдвиг в область низких частот) слоев. Наконец, слабый сдвиг частот ДУНТ $(12,8) @(16,14)$ должен указывать либо на то, что равновесное межслойное расстояние в двустенных нанотрубках меньше, чем $0.34 \mathrm{~nm}$, либо на то, что зависимость $G$-мод от давления в ДУНТ имеет несимметричный характер и зависит от знака (положительного/отрицательного).

\section{4. Заключение}

Исследованы тангенциальные $G$-моды индивидуальных структурно-идентифицированных полупроводниковых двустенных углеродных нанотрубок в зависимости от межслойного расстояния. Независимое определение структурных параметров ДУНТ методами электронной дифракции и просвечивающей электронной микроскопии позволило нам однозначно интерпретировать наблюдаемые особенности спектров КРС двустенных нанотрубок. В спектрах комбинационного рассеяния ДУНТ наблюдается существенный сдвиг тангенциальных мод в сравнении с аналогичными модами одностенных нанотрубок. Показано, что величина сдвига зависит от межслойного расстояния и от величины эффективного давления внутри двустенной нанотрубки.

\section{Список литературы}

[1] C. Shen, A.H. Brozena, Y. Wang. Nanoscale 3, 503 (2011).

[2] K. Fujisawa, H. Kim, S. Go, H. Muramatsu, T. Hayashi, M. Endo, T. Hirschmann, M. Dresselhaus, Y. Kim, P. Araujo. Appl. Sci. 6, 109 (2016).

[3] K.E. Moore, D.D. Tune, B.S. Flavel. Adv. Mater. 27, 3105 (2015).

[4] F. Bonaccorso, P.P.P.-H. Tan, A.C.A. Ferrari, C.G. Centre, J.J.T. Avenue, C. Cb, U. Kingdom, C. Academy. ACS Nano 7, 1838 (2013).

[5] A.M. Rao, P.C. Eklund, S. Bandow, A. Thess, R.E. Smalley. Nature 388, 257 (1997).

[6] D.I. Levshov, Y.I. Yuzyuk, T. Michel, C. Voisin, L. Alvarez, S. Berger, P. Roussignol, J.-L. Sauvajol. J. Phys. Chem. C 114, 16210 (2010).

[7] S. Lebedkin, K. Arnold, O. Kiowski, F. Hennrich, M. Kappes. Phys. Rev. B 73, 094109 (2006).

[8] K.T. Nguyen, A. Gaur, M. Shim. Phys. Rev. Lett. 98, 4 (2007).
[9] M. Paillet, T. Michel, J.C. Meyer, V.N. Popov, L. Henrard, S. Roth, J.L. Sauvajol. Phys. Rev. Lett. 96, 1 (2006).

[10] H. Telg, J.G. Duque, M. Staiger, X. Tu, F. Hennrich, M.M. Kappes, M. Zheng, J. Maultzsch, C. Thomsen, S.K. Doorn. ACS Nano 6, 904 (2012).

[11] F. Villalpando-Paez, H. Muramatsu, Y.A. Kim, H. Farhat, M. Endo, M. Terrones, M.S. Dresselhaus. Nanoscale 2, 406 (2010).

[12] T.C. Hirschmann, P.T. Araujo, H. Muramatsu, J.F. RodriguezNieva, M. Seifert, K. Nielsch, Y.A. Kim, M.S. Dresselhaus. ACS Nano 8, 1330 (2014).

[13] F. Villalpando-Paez, H. Son, D. Nezich, Y.P. Hsieh, J. Kong, Y.a Kim, D. Shimamoto, H. Muramatsu, T. Hayashi, M. Endo, M. Terrones, M.S. Dresselhaus. Nano Lett. 8, 3879 (2008).

[14] T.X. Tinh, N. Van Chuc, V. Jourdain, M. Paillet, D.-Y. Kim, J.-L. Sauvajol, N.T.T. Tam, P.N. Minh. J. Exp. Nanosci. 6, 547 (2011).

[15] R. Arenal, P. Löthman, M. Picher, T. Than, M. Paillet, V. Jourdain. J. Phys. Chem. C 116, 14103 (2012).

[16] D. Levshov, T.X. Than, R. Arenal, V.N. Popov, R. Parret, M. Paillet, V. Jourdain, A.A. Zahab, T. Michel, Y.I. Yuzyuk, J.-L. Sauvajol. Nano Lett. 11,4800 (2011).

[17] D. Levshov, T. Michel, T. Than, M. Paillet, R. Arenal, V. Jourdain, Y.I. Yuzyuk, J.-L. Sauvajol. J. Nanoelectron. Optoelectron. 8,9 (2013).

[18] R. Parret, D. Levshov, T.X. Than, D. Nakabayashi, T. Michel, M. Paillet, R. Arenal, V.N. Popov, V. Jourdain, Y.I. Yuzyuk, A.A. Zahab, J.-L. Sauvajol. MRS Proc. 1407, 11 (2012).

[19] D. Levshov, T. Michel, M. Paillet, X.T. Than, H.N. Tran, R. Arenal, A. Rahmani, M. Boutahir, A.A. Zahab, J.-L. Sauvajol. MRS Proc. 1700, 69 (2014).

[20] D.I. Levshov, M.V. Avramenko, X. Than, T. Michel, R. Arenal, M. Paillet, D.V. Rybkovskiy, A.V. Osadchy, S.B. Rochal, Y.I. Yuzyuk, J. Sauvajol, J. Nanophotonics 10,012 502 (2016).

[21] D.I. Levshov, T. Michel, R. Arenal, H.N. Tran, T.X. Than, M. Paillet, Y.I. Yuzyuk, J.-L. Sauvajol. J. Phys. Chem. C 119, 23196 (2015).

[22] C.S. Allen, C. Zhang, G. Burnell, A.P. Brown, J. Robertson, B.J. Hickey. Carbon. N.Y. 49, 4961 (2011).

[23] V.N. Popov, P. Lambin. Phys. Rev. B 73, 1 (2006).

[24] T. Michel, M. Paillet, A. Zahab, D. Nakabayashi, V. Jourdain, R. Parret, J.-L. Sauvajol. Adv. Nature Sci. Nanosci. Nanotechnol. 1, 045007 (2010).

[25] K. Liu, X. Hong, M. Wu, F. Xiao, W. Wang, X. Bai, J.W. Ager, S. Aloni, A. Zettl, E. Wang, F. Wang. Nature Commun. 4, 1375 (2013).

[26] T. Michel, M. Paillet, A.A. Zahab, D. Nakabayashi, V. Jourdain, R. Parret, J.-L. Sauvajol. Adv. Nature Sci. Nanosci. Nanotechnol. 1, 045007 (2011).

[27] M. Hanfland, H. Beister, K. Syassen. Phys. Rev. B 39, 12598 (1989).

[28] D. Christofilos, J. Arvanitidis, G.A. Kourouklis, S. Ves, T. Takenobu, Y. Iwasa, H. Kataura. Phys. Rev. B 76 (2007). 